\title{
Análise de indicadores de risco para hipertensão arterial em crianças e adolescentes"
}

\author{
ANALYSIS OF RISK INDICATORS FOR THE ARTERIAL HYPERTENSION \\ IN CHILDREN AND TEENAGERS
}

\section{ANÁLISIS DE INDICADORES DE RIESGO PARA LA HIPERTENSIÓN ARTERIAL EN NIÑOS Y ADOLESCENTES}

\section{Thelma Leite de Araújo', Marcos Venícius de Oliveira Lopes², Tahissa Frota Cavalcante ${ }^{3}$, Nirla Gomes Guedes ${ }^{4}$, Rafaella Pessoa Moreira ${ }^{5}$, Emília Soares Chaves ${ }^{6}$, Viviane Martins da Silva ${ }^{7}$}

\section{RESUMO}

O objetivo da pesquisa foi avaliar os indicadores de risco para a hipertensão arterial em crianças e adolescentes. Estudo transversal desenvolvido com 342 indivíduos de seis a 18 anos de uma escola da cidade de Fortaleza (CE). A maioria eram homens (51,5\%). A média de idade foi 11,73 anos $( \pm 3,19)$. Foram freqüentes os escolares com sobrepeso / obesidade $(16,8 \%)$ e com pressão arterial acima do percentil 90 (44,7\%). Sedentarismo, tabagismo e etilismo estiveram presentes em $51,5 \%, 38 \%$ e $15,5 \%$ dos avaliados. A pressão arterial sistólica esteve correlacionada com as variáveis: idade, peso, estatura, perímetros da cintura e do quadril, prega subescapular e Índice de Massa Corporal. Houve correlação da pressão arterial diastólica com idade, peso, estatura, perímetros da cintura e do quadril. Confirma-se a influência de fatores de risco sobre os valores da pressão arterial em jovens. A pressão arterial sistólica foi especialmente influenciada por indicadores antropométricos.

\begin{abstract}
The aim of this study was to evaluate the risk indicators for high blood pressure in children and teenagers. A cross-sectional study developed with 342 individuals of 618 years of age in a school of Fortaleza city. The most individuals were male $(51,5 \%)$. The age mean was 11,7 years $( \pm 3,19)$. It was frequent the scholars with overweight / obesity $(16,8 \%)$ and blood pressure above of the 90 th percentile $(44,7 \%)$. Sedentarism, smoking and drinking were present in $51,5 \%, 38 \%$ and $15,5 \%$ of the sample. The systolic blood pressure was correlated with the variable age, weight, height, waist and hip circumferences, subscapular skinfold thickness and body mass index. There was correlation of the diastolic blood pressure with age, weight, height, and waist and hip circumferences. It was confirm the influence of risk factors on the blood pressure values of children and teenagers. The systolic blood pressure was especially influenced by anthropometric indicators.
\end{abstract}

\begin{abstract}
RESUMEN
El objetivo de la investigación fue evaluar los indicadores de riesgo para la hipertensión arterial en niños y adolescentes. Se trata de un estudio transversal desarrollado con 342 individuos de seis a dieciocho años de una escuela de la ciudad de Fortaleza (CE). La mayoría eran hombres $(51,5 \%)$. El promedio de edad fue de 11,73 años $( \pm 3,19)$. Fueron frecuentes los escolares con sobre peso / obesidad $(16,8 \%)$ y con presión arterial encima del percentil 90 (44,7\%). Sedentarismo, tabaquismo y etilismo estuvieron presentes en el $51,5 \%, 38 \%$ y $15,5 \%$ de los evaluados. La presión arterial sistólica presentó correlación con las variables: edad, peso, estatura, perímetros de la cintura y de la cadera, pliegue subescapular e Índice de Masa Corporal. Se identificó correlación de la presión arterial diastólica con la edad, peso, estatura, perímetros de la cintura y de la cadera. Se confirma la influencia de factores de riesgo sobre los valores de la presión arterial en jóvenes. La presión arterial sistólica estuvo especialmente influenciada por indicadores antropométricos.
\end{abstract}

\section{KEY WORDS \\ Hypertension. Risk Factors. Child. Adolescent.}

\section{DESCRIPTORES \\ Hipertensión. \\ Factores de Riesgo. \\ Niño. \\ Adolescente.}

\footnotetext{
* Fonte de financiamento: Conselho Nacional de Pesquisa e Desenvolvimento Científico e Tecnológico - CNPq no 500639/2003-5; Ministério da Saúde / DECIT / SESA / CEARÁ ${ }^{1}$ Doutora em Enfermagem. Professora Adjunta do Departamento de Enfermagem da Universidade Federal do Ceará. Fortaleza, CE, Brasil. Pesquisadora CNPq. thelmaaraujo2003@yahoo.com.br ${ }^{2}$ Doutor em Enfermagem. Professor Adjunto do Departamento de Enfermagem da Universidade Federal do Ceará. Fortaleza, CE, Brasil. Pesquisador CNPq. marcos@ufc.br ${ }^{3}$ Enfermeira. Mestranda em Enfermagem do Programa de PósGraduação em Enfermagem da Universidade Federal do Ceará. Fortaleza, CE, Brasil. Bolsista do CNPq-Brasil. tahissa@ig.com.br ${ }^{4}$ Enfermeira. Mestranda em Enfermagem do Programa de Pós-Graduação em Enfermagem da Universidade Federal do Ceará. Fortaleza, CE, Brasil. nirlagomes@hotmail.com ${ }^{5}$ Enfermeira. Mestranda em Enfermagem do Programa de Pós-Graduação em Enfermagem da Universidade Federal do Ceará. Fortaleza, CE, Brasil. Bolsista do CNPq-Brasil. rafaellapessoa@hotmail.com ${ }^{6}$ Doutoranda em Enfermagem do Programa de Pós-Graduação em Enfermagem da Universidade Federal do Ceará. Fortaleza, CE, Brasil. Professora da Faculdade Católica Rainha do Sertão. emiliasoareschaves@yahoo.com.br ${ }^{7}$ Doutoranda em Enfermagem do Programa de Pós-Graduação em Enfermagem da Universidade Federal do Ceará. Fortaleza, CE, Brasil. Professora da FaculdadeCatólica Rainha do Sertão. vivianemartinsdasilva@hotmail.com
} 


\section{INTRODUÇÃO}

A hipertensão arterial apresenta alta prevalência na população brasileira, influenciando na gênese de doenças cerebrovasculares, coronarianas, doença de retina, insuficiência cardíaca, insuficiência renal crônica e doenças vasculares ${ }^{(1)}$. O reconhecimento do aumento da prevalência da hipertensão arterial na população jovem e de suas possíveis complicações na vida adulta tem implicações importantes para a prevenção de doenças crônicas, em especial as cardiovasculares.

Ações de promoção da saúde relacionadas com mudanças de estilo de vida representam a possibilidade de prevenção mais efetiva da ocorrência de eventos cardiovasculares. Os estudos relativos à detecção dos indicadores de risco em populações jovens são essenciais para o acompanhamento dos indivíduos que apresentam maior risco de alterações na idade adulta.

Entre os diversos indicadores de risco que contribuem para o desenvolvimento da hipertensão arterial em crianças e adolescentes, destacamse: os níveis iniciais elevados de pressão arterial, a história familiar, a obesidade, o sedentarismo, o tabagismo e o etilismo. Estudos longitudinais têm demonstrado que crianças com níveis de pressão arterial elevados apresentam maior probabilidade de se tornarem adultos portadores de hipertensão arterial $\left.\right|^{(2-3)}$. Um estudo ${ }^{(4)}$ mostrou que os valores iniciais altos durante a infância estiveram correlacionados positivamente com os valores da pressão arterial sistólica e diastó-lica quatro anos mais tarde.

Outros estudos indicaram uma associação positiva entre o Índice de Massa Corporal elevado e a prevalência da hipertensão arterial|(5-6). $O$ excesso de massa corporal é um fator predisponente para a hipertensão, podendo ser responsável por $20 \%$ a $30 \%$ dos casos de hipertensão arterial $\left.\right|^{(1)}$. Por outro lado, estudos em crianças e adolescentes que correlacionam outros indicadores antropometricos (pregas cutâneas, perímetro da cintura e a relação cintura/quadril) com os valores da pressão arterial são escassos.

Além da diminuição do peso corpóreo, o controle da pressão arterial está relacionado à prática regular de atividades físicas. Um estudo de meta-análise ${ }^{(7)}$ concluiu que o exercício físico aeróbico, contribuiu para uma redução de $3 \mathrm{mmHg}$ da pressão arterial sistólica e diastólica em indivíduos normotensos, $6 \mathrm{mmHg}$ da pressão arterial sistólica e $7 \mathrm{mmHg}$ da pressão arterial diastólica em hipertensos limítrofes, e $10 \mathrm{mmHg}$ da pressão sistólica e $8 \mathrm{mmHg}$ da diastólica em indivíduos com hipertensão severa.

Em relação aos efeitos diretos do tabagismo, há evidências demonstrando haver uma leve obstrução nas vias aéreas e retardo de crescimento da função pulmonar em adolescentes. Ademais, a exposição passiva ao fumo está relacionada a baixos níveis plasmáticos de HDL associado a uma disfunção endotelial (vasoconstrição) significativa dose-dependente ${ }^{(8)}$.

Por outro lado, estudos que correlacionam a pressão arterial de crianças e adolescentes com o etilismo são raros. É conhecido que a ingesta elevada e crônica do álcool, na população adulta, pode contribuir para o desenvolvimento de hepáticas (esteatose hepática, hepatite alcoólica e cirrose), digestivas (gastrite, síndrome de má absorção e pancreatite) e cardiovasculares (hipertensão) ${ }^{(9)}$.

Daí, existe carência de estudos em crianças e adolescentes que relacionem os diversos indicadores antropométricos, etilismo, tabagismo e prática de exercícios físicos com os valores da pressão arterial. A partir deste contexto, o propósito do presente estudo foi avaliar esse conjunto de indicadores de risco para a hipertensão arterial na população infanto-juvenil, buscando identificar correlações com os valores da pressão arterial.

\section{MÉTODO}

\section{Desenho}

Estudo transversal, desenvolvido numa escola de ensino fundamental e médio da cidade de Fortaleza-Ceará / Brasil, nos períodos de outubro de 2004 a março de 2005 e junho a outubro de 2005 . A população foi composta por crianças e adolescentes de 6 a 18 anos matriculados na escola nos turnos da manhã e da tarde, que totalizavam 385 escolares.

Buscou-se captar todas as crianças e adolescentes, porém houve a perda de 43 escolares devido a não aceitação de participar do estudo ou a saída da escola no período da coleta de dados. Assim, a amostra foi composta por 342 crianças e adolescentes que aceitaram participar do estudo, após a autorização assinada pelos pais.

\section{Coleta dos dados}

Para a coleta de dados foi utilizado um formulário específico que contém tópicos que possibilitaram atingir os objetivos propostos. As variáveis investigadas no estudo foram: sexo, idade, história familiar para alterações cardiovasculares, tipo e freqüência de atividades físicas, hábito de fumar e de consumir bebidas alcoólicas, altura, peso corporal, pregas cutâneas, circunferência da cintura, do quadril e do membro utilizado para a verificação da pressão arterial, tamanho do manguito utilizado e valores da pressão arterial sistólica e diastólica. Foram realizadas três medidas de cada prega cutânea e da pressão arterial, sendo calculadas suas médias. 
Nas medidas da pressão arterial, foram adotadas as recomendações internacionais que apresentam diretrizes para os protocolos de medida da pressão arterial ${ }^{(10)}$. Foram utilizados os seguintes materiais: esfigmomanômetros com manômetros aneróides devidamente testados e calibrados, estetoscópios duplos e manguitos de larguras correspondentes a $40 \%$ da circunferência do braço utilizado para a verificação da pressão arterial.

A prática anterior de atividades físicas, esvaziamento da bexiga e o tempo decorrido desde a última alimentação foram investigados antes da verificação da pressão arterial. As crianças e os adolescentes permaneceram sentados em um ambiente tranqüilo e silencioso por, no mínimo, cinco minutos. A pressão arterial foi verificada sempre no braço direito, três vezes com intervalos de um minuto entre cada verificação e calculada média aritmética, sendo esse o valor considerado para a análise. $\mathrm{Na}$ ocorrência de diferença igual ou superior a $6 \mathrm{mmHg}$ entre os valores da PAS e/ou PAD, era realizada nova verificação da pressão arterial e calculada a média dos três valores de PAS e/ou PAD mais próximos, sendo excluído o valor mais divergente. A pressão arterial sistólica foi determinada pelo aparecimento do som de Korotkoff (KI) e a pressão arterial diastólica pelo desaparecimento do som de Korotkoff $(\mathrm{KV})^{(1)}$.

Para a coleta de dados antropométricos (peso, estatura, pregas cutâneas tricipital e subescapular, além do perímetro da cintura e do quadril) foram utilizados os seguintes equipamentos: balança antropométrica devidamente testada e calibrada da marca Plenna Futura Digital, com capacidade de $150 \mathrm{Kg}$ e precisão de $100 \mathrm{~g}$, haste de balança antropométrica, com capacidade de $2 \mathrm{~m}$ e sensibilidade de $0,5 \mathrm{~cm}$, fita métrica não distensível com intervalos de $0,1 \mathrm{~cm}$ e extensão de $100 \mathrm{~cm}$ e adipômetro da marca Sanny, com precisão de $1 \mathrm{~mm}$ e capacidade de 58 $\mathrm{mm}$. As medidas foram verificadas três vezes e calculada a média.

As medidas do peso foram realizadas com o participante em posição ortostática, posicionado no centro da balança com os pés juntos e os braços estendidos ao longo do corpo. A estatura foi verificada com a haste embutida na balança antropométrica. O participante encontrava-se na posição ortostática, pés descalços e unidos, braços pendentes, com as mãos espalmadas sobre as coxas e queixo ereto.

O perímetro da cintura foi mensurado com fita métrica colocada horizontalmente no ponto médio entre a borda inferior da última costela e a crista ilíaca, sendo a leitura realizada entre uma expiração e uma inspiração. Para medida do perímetro do quadril, a fita métrica foi colocada horizontalmente em torno do quadril na parte mais saliente dos glúteos.
Após o cálculo do valor médio da pressão arterial, a interpretação dos valores pressóricos das crianças e adolescentes foi realizada, utilizando como referência, uma tabela de percentis ${ }^{(11)}$ que, classifica os níveis de pressão arterial em: normal (menor que o percentil 90); pré-hipertensão (igual ao percentil 90 e menor que o percentil 95); hipertensão estágio 1 (entre o percentil 95 e o percentil 99 mais $5 \mathrm{~mm} \mathrm{Hg}$ ); hipertensão estágio 2 (acima de $5 \mathrm{~mm} \mathrm{Hg}$ do percentil 99). No estudo foram considerados como portadores de valores alterados de pressão arterial aqueles participantes que apresentaram valores iguais ou acima do percentil 90. Na análise da prática de atividades físicas foram considerados como sedentários os indivíduos que praticavam atividades físicas com freqüência menor que três vezes por semana e duração menor de 20 minutos em cada vez.

Os valores obtidos no Índice de Massa Corporal foram classificados em percentis de acordo com o inquérito americano National Center for Health Statistics ${ }^{(12)}$, como: baixo peso (abaixo do percentil 5); normal (entre o percentil 5 e 85); sobrepeso (entre o percentil 85 e 95); obesidade (acima do percentil 95). Na análise do tabagismo foram considerados fumantes, aqueles que apresentavam hábito ativo de fumar e / ou hábito passivo (pais, irmãos ou amigos fumantes).

\section{Análise dos dados}

Para verificação da normalidade dos dados utilizouse o teste de Kolmogorov-Smirnov. Para verificação de homocedasticidade utilizou-se o teste de Levene. Na análise de diferenças de média utilizou-se o teste $T$, supondo variâncias iguais quando os princípios de normalidade e homocedasticidade foram respeitados. Na identificação de heterocedasticidade, utilizou-se o Teste $T$ supondo variâncias desiguais. Para análise de correlação utilizou-se o coeficiente de correlação de Pearson quando as variáveis apresentavam linearidade, homocedasticidade e normalidade. Na ausência de uma destas características, utilizou-se o coeficiente de correlação de Spermann. Os dados foram processados e analisados com auxílio do Software SPSS versão 13.0. O nível de significância adotado foi de $5 \%(p<0,05)$.

\section{Considerações éticas}

O estudo foi autorizado pela direção da escola. Ademais, a proposta foi encaminhada e aprovada por Comitê de Ética em Pesquisa, protocolada sob n. 58/03. A coleta de dados iniciou com as devidas aprovações e após reunião com os pais dos alunos, onde foram esclarecidos sobre os objetivos do estudo e solicitada a autorização por escrito, com a assinatura do Termo de Consentimento Livre e Esclarecido. 


\section{RESULTADOS}

Tabela 1 - Diferenças de média entre a pressão arterial sistólica e a pressão arterial diastólica em crianças e adolescentes, por sexo, atividade física, tabagismo e etilismo $(\mathrm{n}=342)$ - Fortaleza -2005

\begin{tabular}{|c|c|c|c|c|c|c|}
\hline & \multicolumn{3}{|c|}{ PAS } & \multicolumn{3}{|c|}{ PAD } \\
\hline & Média $( \pm \mathrm{SD})$ & $\mathrm{SE}$ & Sig. & Média $( \pm \mathrm{SD})$ & $\mathrm{SE}$ & Sig. \\
\hline \multicolumn{7}{|l|}{ Sexo } \\
\hline Masculino & $110,91( \pm 10,41)$ & 0,78 & $\mathrm{p}=0,681^{*}$ & $73,12( \pm 10,22)$ & 0,77 & $\mathrm{p}=0,084^{*}$ \\
\hline Feminino & $108,22( \pm 10,41)$ & 0,80 & $\mathrm{p}=0,016 \dagger$ & $73,47( \pm 7,93)$ & 0,62 & $\mathrm{p}=0,752 \dagger$ \\
\hline \multicolumn{7}{|l|}{ Atividade física } \\
\hline Adequada & $110,35( \pm 9,82)$ & & $\mathrm{p}=0,203^{*}$ & $73,45( \pm 9,93)$ & & $\mathrm{p}=0,230^{*}$ \\
\hline Inadequada & $109,27( \pm 11,56)$ & & $\mathrm{p}=0,318^{\ddagger}$ & $73,28( \pm 8,49)$ & & $\mathrm{p}=0,830^{\ddagger}$ \\
\hline \multicolumn{7}{|l|}{ Fumante } \\
\hline $\operatorname{Sim}$ & $108,78( \pm 10,47)$ & & $\mathrm{p}=0,753^{*}$ & $73,20( \pm 7,75)$ & & $\mathrm{p}=0,051^{*}$ \\
\hline Não & $110,45( \pm 10,50)$ & & $\mathrm{p}=0,377^{\star}$ & $73,67( \pm 8,93)$ & & $\mathrm{p}=0,064^{+}$ \\
\hline \multicolumn{7}{|c|}{ Uso de bebidas alcoólicas } \\
\hline Sim & $109,45( \pm 8,63)$ & & $\mathrm{p}=0,010^{*}$ & $72,61( \pm 8,74)$ & & $\mathrm{p}=0,803^{*}$ \\
\hline Não & $109,69( \pm 10,84)$ & & $\mathrm{p}=0,843^{*}$ & $73,65( \pm 8,41)$ & & $\mathrm{p}=0,441^{\ddagger}$ \\
\hline
\end{tabular}

* Teste de Levene. †Teste T assumindo variâncias iguais. $¥$ Teste T assumindo variâncias desiguais.

A maioria dos indivíduos era do sexo masculino $(51,5 \%)$ com média de idade de 11,73 anos $( \pm 3,19)$. As crianças e os adolescentes do sexo masculino apresentaram médias da pressão arterial sistólica maiores do que as do sexo feminino $(p=0,016)$.
As médias do peso, altura, perímetro da cintura, da relação cintura-quadril, da prega tricipital e da prega subescapular foram $46,10 \mathrm{~kg}( \pm 18,90), 1,48 \mathrm{~cm}( \pm 0,89)$, $66,42 \mathrm{~cm}( \pm 10,64), 0,81 \mathrm{~cm}( \pm 0,59), 11,42 \mathrm{~mm}( \pm 4,70)$ e $10,00 \mathrm{~mm}( \pm 5,00)$, respectivamente.

Tabela 2 - Distribuição dos indicadores de risco para a hipertensão arterial das crianças e adolescentes - Fortaleza - 2005

\begin{tabular}{|c|c|c|c|c|}
\hline Indicadores de risco & Sim & Não & Não sabe & Total \\
\hline Alteração inicial na pressão arterial & $153(44,7 \%)$ & $189(55,3 \%)$ & - & $342(100 \%)$ \\
\hline História familiar ${ }^{*}$ & $190(55,6 \%)$ & $85(24,8 \%)$ & $67(19,6 \%)$ & $342(100 \%)$ \\
\hline Sobrepeso/Obesidade & $58(16,8 \%)$ & $284(83,2 \%)$ & - & $342(100 \%)$ \\
\hline Sedentarismo & $176(51,5 \%)$ & $166(48,5 \%)$ & - & $342(100 \%)$ \\
\hline Tabagismo & $130(38 \%)$ & $212(62 \%)$ & - & $342(100 \%)$ \\
\hline
\end{tabular}

* Obtida pela informação da criança ou do adolescente.

Foi identificada uma alta freqüência de pressão arterial igual ou acima do percentil $90(44,7 \%)$. Mais da metade do grupo participante $(55,6 \%)$ referiu história familiar, até segundo grau, de hipertensão arterial. Encontrou-se que $16,8 \%$ dos indivíduos apresentaram sobrepeso ou obesidade e $51,5 \%$ eram sedentários. Verificou-se que $38 \%$ das crianças e adolescentes tinham hábitos tabagistas, sendo $36 \%$ consideradas fumantes passivos. Houve baixa freqüência de ingestão de bebidas alcoólicas nas crianças e adolescentes (15,5\%). 
Tabela 3 - Correlação entre as pregas cutâneas, peso, altura, IMC, perímetro da cintura e do quadril, RCQ e idade, com a PAS e a PAD (n=342) - Fortaleza - 2005

\begin{tabular}{|c|c|c|c|c|}
\hline \multirow{2}{*}{ Variáveis } & \multicolumn{2}{|c|}{ PAS } & \multicolumn{2}{|c|}{ PAD } \\
\hline & $\mathrm{R}$ & Sig & $\mathrm{R}$ & Sig \\
\hline Idade & $0,352^{*}$ & 0,000 & $0,204^{*}$ & 0,000 \\
\hline Prega Tricipital & $-0,141^{*}$ & 0,795 & $-0,306^{*}$ & 0,091 \\
\hline Prega Subescapular & $0,165^{*}$ & 0,003 & $0,580^{*}$ & 0,093 \\
\hline Perímetro da Cintura & $0,280^{*}$ & 0,000 & $0,110^{*}$ & 0,028 \\
\hline Perímetro do Quadril & $0,343^{* *}$ & 0,000 & $0,173^{* *}$ & 0,001 \\
\hline Razão Cintura/Quadril & $-0,138^{*}$ & 0,011 & $-0,172^{*}$ & 0,001 \\
\hline Peso & $0,301^{* *}$ & 0,000 & $0,172^{* *}$ & 0,002 \\
\hline Altura & $0,436^{*}$ & 0,000 & $0,263^{*}$ & 0,000 \\
\hline IMC & $0,207^{*}$ & 0,000 & $0,084^{*}$ & 0,132 \\
\hline
\end{tabular}

* Coeficiente de correlação de Spearman; ** Coeficiente de Correlação de Pearson.

Na análise de correlação entre a pressão arterial e as medidas antropométricas, a pressão arterial sistólica apresentou correlação positiva estatisticamente significante com a idade $(p=0,000)$, a prega subescapular $(p=$ $0,003)$, os perímetros da cintura $(p=0,000)$ e do quadril ( $p$ $=0,000)$, o peso $(p=0,000)$, a altura $(p=0,000)$ e o IMC (p $=0,000)$.

A pressão arterial diastólica apresentou correlacionada positiva estatisticamente significante com idade ( $p$ $=0,000)$, perímetros da cintura $(p=0,028)$ e do quadril ( $p$ $=0,001)$, peso $(p=0,002)$ e altura $(p=0,000)$. A RCQ apresentou correlação negativa com a pressão arterial sistólica $(p=0,011)$ e a pressão arterial diastólica ( $p=$ 0,001 ). Não foi identificada diferença de média entre a pressão arterial sistólica e diastólica com a prática de atividade física, tabagismo e etilismo.

\section{DISCUSSÃO}

A prevalência da hipertensão arterial tem aumentado na população infanto-juvenil, no qual seu valor varia de 2 a $13 \%{ }^{(1)}$. No presente estudo identificamos $44,7 \%$ de crianças e adolescentes com pressão arterial inicial igual ou acima do percentil 90. Este número é superior ao encontrado em um estudo(2) que teve como achado uma prevalência de $16,2 \%$ da pressão arterial sistólica ou diastólica acima do percentil 90 em 1005 crianças e adolescentes. Ademais, um outro estudo(13) identificou uma prevalência ainda menor da ordem de 7,7\% em escolares de 7 a 17 anos com a pressão arterial igual ou acima do percentil 95.

A ocorrência mais freqüente de alterações na pressão arterial pode ser explicada pelo fato de que as crianças e adolescentes foram avaliados em uma única visita. Alguns autores $^{(2)}$ justificam que taxas mais elevadas são encontradas em estudos que se baseiam em uma avaliação, e que os valores caem significativamente quando são considerados portadores de pressão arterial elevada, aqueles que têm a pressão arterial igual ou acima do percentil 90 em duas ou três avaliações consecutivas.
Os antecedentes familiares para a hipertensão arterial foram identificados em $55,6 \%$ dos indivíduos avaliados. Fortes evidências sugerem que a história familiar de hipertensão arterial é um dos maiores fatores na determinação dos níveis de pressão arterial ${ }^{(3)}$. Ao estudarem a influência da hereditariedade na origem da hipertensão, alguns pesquisadores referem que os componentes de uma mesma família, além de partilharem entre si os genes, compartilham também o mesmo ambiente cultural e doméstico ${ }^{(14)}$.

A presença do indicador de risco história familiar de hipertensão determina maior risco para que os filhos também desenvolvam hipertensão arterial. Isso foi confirmado por um estudo realizado em São Paulo(15), no qual os valores da pressão arterial sistólica e diastólica foram maiores para filhos de hipertensos do que para os filhos de normotensos.

Com relação ao sobrepeso / obesidade nossos achados se aproximam aos de outro estudo ${ }^{(6)}$ no qual $17,7 \%$ dos indivíduos entre 2 e 11 anos apresentavam alterações do peso. Outra investigação realizada com adolescentes obesos $^{(16)}$ encontrou um incremento na pressão arterial sistólica e na pressão arterial diastólica de $10 \mathrm{mmHg}$ e $4 \mathrm{mmHg}$, respectivamente quando comparados aos padrões esperados. Foi constatado também por um estudo ${ }^{(5)}$ que a chance de um indivíduo com obesidade ser também portador de hipertensão arterial é 7,53 vezes maior que a chance de um indivíduo com sobrepeso. Comparando indivíduos com sobrepeso e indivíduos com peso normal, o risco de desenvolver hipertensão aumenta em $180 \%^{(17)}$. Diante disso, o incentivo para a redução do peso deve ser considerado prioritário, pois até mesmo pequenas perdas podem resultar em significativa queda da pressão arterial.

Em relação ao sedentarismo, 51,5\% dos avaliados praticavam exercícios físicos menos que três vezes por semana e / ou com duração inferior a vinte minutos em cada vez. Evidências indicam que a prática de atividade física regular oferece benefícios diretos e indiretos que auxiliam a redução da pressão arterial e do risco cardiovascular total|(7). 
$\mathrm{O}$ alto percentual de fumantes passivos identificado em nosso estudo (36\%) pode contribuir para o desenvolvimento de enfermidades a curto e longo prazo. As crianças fumantes passivas têm uma maior freqüência de resfriados, infecções do ouvido médio e de doenças respiratórias, tais como a pneumonia, bronquites e exacerbação da asma ${ }^{(18)}$. Em adultos, existe o risco $30 \%$ maior de câncer de pulmão e $24 \%$ maior de infarto do miocárdio do que os não-fumantes que não se expõem ao tabaco ${ }^{(18)}$. Houve uma baixa freqüência de ingestão de bebidas alcoólicas nas crianças e adolescentes (15,5\%). Não foram encontrados estudos sobre a influência de bebidas alcoólicas nos valores da pressão arterial de jovens.

A diferença de média da pressão arterial sistólica por sexo identificada em nosso estudo foi semelhante à encontrada por um estudo ${ }^{(19)}$, onde a PAS foi significativamente maior em homens. Por outro lado, sabe-se que até os 12 anos de idade, a pressão arterial sistólica é semeIhante para ambos os sexos, ou mesmo mais alta no sexo feminino, ocorrendo uma inversão desses valores no final da adolescência.

Com relação à influência das medidas antropométricas e os valores da pressão arterial, a literatura relata haver uma correlação positiva entre a pressão arterial e a idade, o peso, a estatura e o Índice de Massa Corporal(4). Estes dados concordam com os encontrados em nosso estudo. Entretanto, alguns autores sugerem que o tamanho corporal (altura e peso) seja mais importante do que a idade ${ }^{(20)}$.

Ademais, um estudo ${ }^{(20)}$ encontrou correlação positiva e significativa da pressão arterial com as pregas cutâneas tricipital e subescapular. Neste estudo, somente foi observada uma correlação significativa entre a pressão arterial sistólica e a prega subescapular. Outros parâmetros de avaliação da gordura central (perímetro da cintura e do quadril) estiveram também correlacionados com a pressão arterial, porém, as pesquisas desenvolvidas sobre a relação entre estas variáveis ainda não são conclusivas.

\section{REFERÊNCIAS}

1. Mion Júnior $D$, coordenador. $V$ Diretrizes Brasileira de Hipertensão Arterial. São Paulo: Sociedade Brasileira de Cardiologia; 2006.

2. Oliveira RG, Lamounier JA, Oliveira ADB, Castro MDR, Oliveira JS. Pressão arterial em escolares e adolescentes: o estudo de Belo Horizonte. J Pediatr. 1999;75(4): 75-81.

3. Fuentes RM, Notkola IL, Shemeikka S, Tuomilehto J, Nissinen A. Tracking of systolic blood pressure during childhood: a 15-year follow-up population-based family study in eastern Finland. J J Hypertens 2002;20(2): 195-202.
Não encontramos diferença de média entre a pressão arterial sistólica e diastólica com a prática de atividade física, tabagismo e etilismo. Por outro lado, um estudo comparativo realizado entre adolescentes fumantes e não fumantes, encontrou uma diferença de $12 \mathrm{mmHg}$ na pressão arterial sistólica e de $5 \mathrm{mmHg}$ da pressão arterial diastólica entre os adolescentes fumantes quando comparados aos não-fumantes ${ }^{(16)}$. As pesquisas indicam que estes indicadores de risco contribuem para a instalação de várias doenças crônicas, em especial as cardiovasculares ${ }^{(8-9)}$.

Apesar das limitações desse estudo, como a avaliação da pressão arterial em visita única e da abrangência da faixa etária (6 a 18 anos), o estudo aponta que alguns parâmetros antropométricos como o peso, a altura e os perímetros da cintura e do quadril parecem ser preditores de alterações da pressão arterial em jovens. Contudo, é necessária a realização de estudos longitudinais que confirmem estes achados.

\section{CONCLUSÕES}

Na classificação dos valores de pressão arterial, segundo os percentis de pressão arterial para o sexo, idade e estatura foi considerável a freqüência de crianças e adolescentes com valores de pressão arterial acima do percentil $90(44,7 \%)$. É válido ressaltar que essas alterações da pressão arterial não indicam que estes indivíduos sejam portadores de hipertensão arterial, mas sugerem uma possível evolução para níveis elevados na vida adulta, sobretudo se houver a presença de fatores de risco e se medidas de orientação não forem implementadas.

As medidas antropométricas apresentaram correlações significativas com os valores da PAS e PAD, notadamente as variáveis idade, peso, estatura, perímetros da cintura e do quadril. A RCQ esteve correlacionada negativa e significativamente com as pressões arteriais sistólica e diastólica. Contudo, é necessária a realização de estudos longitudinais que confirmem estes achados.

4. Li L, Wang Y, Cao W, Xu F, Cao J. Longitudinal studies of blood pressure in children. Asia Pac J Public Health 1995; $8(2): 130-3$.

5. Carneiro G, Faria NA, Barreto-Filho FFR, Guimarães A, Lerário $D$, Ferreira SRG, et al. Influência da distribuição da gordura corporal sobre a prevalência de hipertensão arterial e outros fatores de risco cardiovasculares em indivíduos obesos. Rev Assoc Med Bras. 2003;49(3): 306-11. 
6. Garcia FD, Terra AF, Queiroz AM, Correia CA, Ramos PS, Ferreira QT, et al. Avaliação de fatores de risco associados com elevação da pressão arterial em crianças. J Pediatr. 2004;80(1):29-34.

7. Fagard R, Amery A. Physical exercise in hypertension. In: Laragh JBB. Hypertension: pathophysiology, diagnosis and management. New York: Reaven Press;1995. p. 2669-79.

8. Gold DR, Wang X, Wypij D, Speizer FE, Ware JH, Dockery DW. Effects of cigarette smoking on lung function in adolescents boys and girls. N Engl J Méd. 1996; 335 (13): 931-7.

9. Weinberger MH. Sódio e outros fatores da dieta. In: Weber MA. Hipertensão. Rio de Janeiro: Guanabara Koogan; 2003. p. 30-4.

10. Perloff D, Grim C, Flack J, Frohlich DE, Hill M, McDonald $M$, et al. Human blood pressure determination by sphymomanometry. Circulation. 1993;88(5 Pt 1):2460-70.

11. National High Blood Pressure Education Program Working Group on High Blood Pressure in Children and Adolescents. The fourth report on the diagnosis, evaluation and treatment of high blood pressure in children and adolescents. Pediatrics. 2004;114(2): 555-76.

12. National Center for Health Statistics (NCHS). CDC Growth Charts: United States. Hyattsville; 2000.

13. Silva MAM, Rivera IR, Ferraz MRMT, Pinheiro AJT, Alves SWS, Moura AA, et al. Prevalência de fatores de risco cardiovascular em crianças e adolescentes da rede de ensino da cidade de Maceió. Arq Bras Cardiol. 2005; 84(5):387-92.
14. Francischetti EA, Fagundes VGA. A história natural da hipertensão essencial começa na infância e adolescência? HiperAtivo. 1996;3(2):77-85.

15. Elias MC, Bolívar MSM, Fonseca FAH, Martinez TLR, Angelini J, Ferreira C, et al. Comparação do perfil lipídico, pressão arterial e aspectos nutricionais em adolescentes, filhos de hipertensos e de normotensos. Arq Bras Cardiol. 2004;82(2):139-42.

16. Ramírez EM, Montero AG, Sol JMM, Paneque RJ, Roque GP. Factores de riesgo asociados con la tensión arterial en adolescentes. Rev Cubana Méd Gen Integr. 2001;17(15):435-40.

17. Haffner SM, Ferrannini E, Hazuda HP, Stern MP. Clutering of cardiovascular risk factors in confirmed pre hypertensive individuals. Hypertension. 1992;20(1):38-45.

18. Brasil. Ministério da Saúde. Instituto Nacional do Câncer. Tabagismo passivo: efeitos da fumaça na saúde das crianças [homepage na Internet]. [citado $2007 \mathrm{fev}$. 28]. Disponível em: http://www.inca.gov.br/tabagismo/ frameset.asp?item=passivo\&link=crianca.htm.

19. Gillman MW, Cook NR, Rosner B, Evans DA, Keough ME, Taylor JO, et al. Identifying children at high risk for the development of essential hypertension. J Pediatr. 1993;122(6):837-46.

20. Mori C, Watanabe K, Haneda N, Harada Y, Zhong ZW, Nishio T. Quantitative analysis of the tracking of blood pressure and relative factors in childhood: Shimane Heart Study. Clin Exp Pharmacol Physiol. 1992;19(20): 69-74. 